\title{
Folic acid deficiency declined substantially after introduction of the mandatory fortification programme in Queensland, Australia: a secondary health data analysis
}

\author{
Anna Slagman ${ }^{1,2, *}$, Linton Harriss ${ }^{1}$, Sandra Campbell ${ }^{1,3}$, Reinhold Muller ${ }^{1}$ and \\ Robyn McDermott ${ }^{1,4}$ \\ 'James Cook University, Medical and Veterinary Sciences, Australian Institute of Tropical Health and Medicine, \\ College of Public Health, Centre for Chronic Disease Prevention, Cairns, Australia: ${ }^{2}$ Notfallmedizinische \\ Versorgungsforschung, Notfall- und Akutmedizin CVK, CCM, Charité Universitätsmedizin Berlin, Charitéplatz 1, \\ 10117 Berlin, Germany: ${ }^{3}$ Central Queensland University, Cairns, Australia: ${ }^{4}$ University of South Australia, Adelaide, \\ Australia
}

Submitted 28 August 2018: Final revision received 10 May 2019: Accepted 22 May 2019: First published online 4 September 2019

\begin{abstract}
Objective: To investigate the prevalence of folic acid deficiency in Queenslandwide data of routine laboratory measurements, especially in high-risk subpopulations.

Design: Secondary health data analysis.

Setting: Analysis of routine folic acid tests conducted by Pathology Queensland (AUSLAB).

Participants: Female and male persons aged 0-117 years with routine folic acid testing between 1 January 2004 and 31 December 2015. If repeat tests on the same person were conducted, only the initial test was analysed ( $n 291908$ ).

Results: Overall the prevalence of folic acid deficiency declined from $7.5 \%$ before (2004-2008) to $1 \cdot 1 \%$ after mandatory folic acid fortification $(2010-2015 ; P<0.001)$ reflecting a relative reduction of $85 \%$. Levels of erythrocyte folate increased significantly from a median (interquartile range) of 820 (580-1180) nmol/l in 2008 before fortification to $1020(780-1350) \mathrm{nmol} / 1$ in $2010(P<0.001)$ after fortification. The prevalence of folic acid deficiency in the Indigenous population (14792 samples) declined by $93 \%(17.4 v .1 .3 \% ; P<0.001)$; and by $84 \%$ in non-Indigenous residents $(7.0$ v. $1.1 \% ; P<0.001)$. In a logistic regression model the observed decrease of folic acid deficiency between 2008 and 2010 was found independent of gender, age and ethnicity $\left(\mathrm{OR}_{\text {crude }}=0.20 ; 95 \%\right.$ CI $0.18,0.23 ; P<0.001$; $\left.\mathrm{OR}_{\text {adjusted }}=0.21 ; 95 \% \mathrm{CI} 0 \cdot 18,0 \cdot 23 ; P<0 \cdot 001\right)$.

Conclusions: While voluntary folic acid fortification, introduced in 1995, failed especially in high-risk subgroups, the 2009 mandatory folic acid fortification programme coincided with a substantial decrease of folic acid deficiency in the entire population.
\end{abstract}

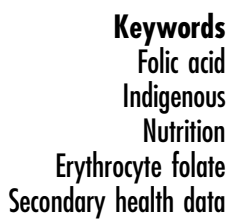

Keywords

Folic acid

ndigenous

yte folate

Secondary health data
Folic acid is an essential micronutrient which is found in fruits and vegetables, nuts, liver and lentils, and has a pivotal role in the human body as a major coenzyme in carbon metabolism $^{(1)}$. Folic acid deficiency has been shown to be associated with neural tube defects (NTD) in newborns but has also been reported to influence the development and progression of other chronic diseases ${ }^{(1)}$. In 1995 a voluntary folic acid fortification programme was introduced in Australia ${ }^{(2)}$. Even though a subsequent increase in folic acid levels as well as a decline in folic acid deficiency ${ }^{(3)}$ and prevalence of $\mathrm{NTD}^{(4,5)}$ were observed, the effects were small compared with the USA, where a mandatory folic acid fortification programme was implemented in $1996^{(6)}$. In particular, high-risk Australian populations (Indigenous populations, persons with low socio-economic status, persons living in remote areas) showed no benefit from voluntary folic acid fortification ${ }^{(7-9)}$.

In September 2009, Australia implemented mandatory folic acid fortification of bread-making flour $(2-3 \mathrm{mg} / \mathrm{kg}$ wheat flour ${ }^{(3,10-12)}$. It was expected that a folic acid intake 


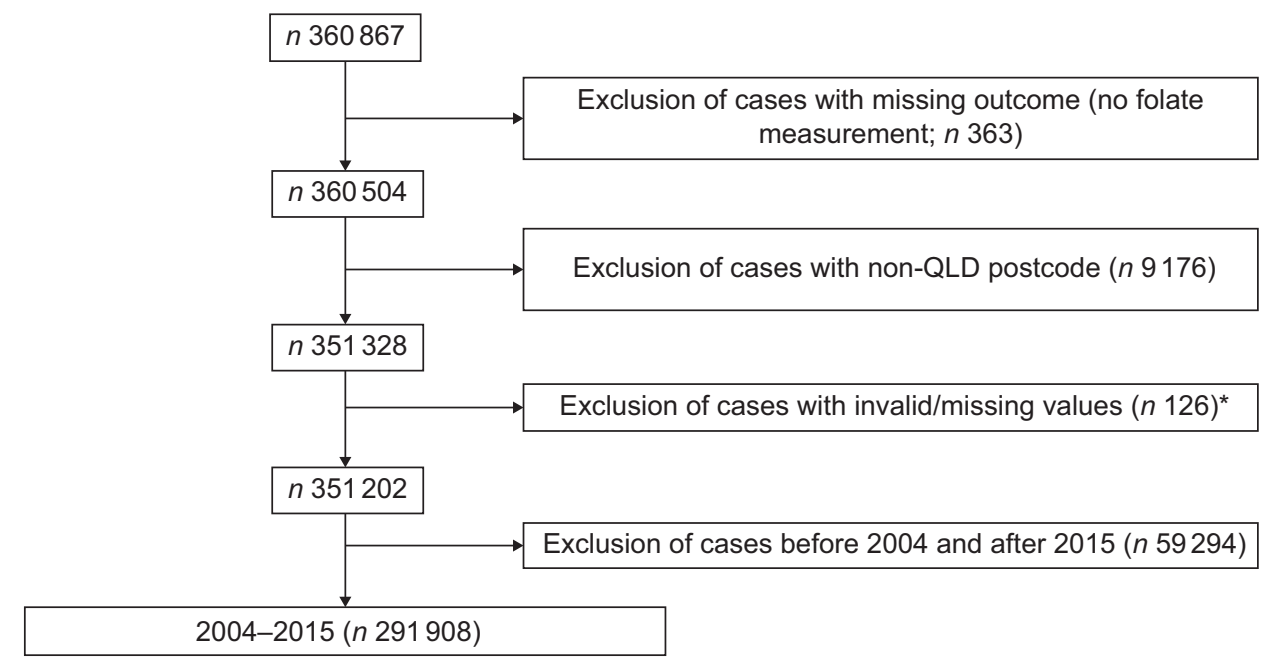

Fig. 1 Selection of data for the current analysis. People with missing outcome information or missing information on any main influencing factors were excluded. Due to incompleteness of some data, measurements before 2004 were excluded. The final data set included 291908 individual measurements. *Serum folate $(n 4)$, invalid age $(n 1)$, missing age $(n 1)$, missing gender $(n 120)$

of $120 \mu \mathrm{g}$ per $100 \mathrm{~g}$ bread consumed would be achieved ${ }^{(13)}$. Evaluations of this programme to date have been confined to small populations within close regional boundaries and have shown a positive impact on folic acid levels and on the occurrence of folic acid deficiency ${ }^{(14-16)}$. Additionally to folic acid deficiency, folic acid insufficiency in women of childbearing age contributes to increased rates of NTD and has not been evaluated so far in Australia ${ }^{(17,18)}$.

Using population pathology data obtained from a Queensland medical laboratory, we undertook a statewide evaluation of mandatory folic acid fortification, assessing folic acid deficiency in high-risk sub-populations such as Indigenous residents, as well as folic acid insufficiency in women of childbearing age.

\section{Methods}

\section{Study design}

The present study is a secondary health data analysis of routinely collected health data from Pathology Queensland (AUSLAB). Data on all folic acid measurements between 1 January 2000 and 31 December 2015 were retrieved from the laboratory information systems and extracted into Excel files for male and female persons aged 0 to 117 years. Where multiple tests per person were identified, only data of the initial test were retained. The data retrieved contained demographic information (date of birth, gender, postcode, ethnicity), date, time and location of folic acid measurement, folic acid values as well as further laboratory findings. Data were transferred into IBM SPSS Statistics version 25 and extensively checked for completeness and validity. In a first step, invalid ( $n$ 1768) and repeated tests ( $n$ 345 489) were excluded from analysis. Of the remaining cases, those with missing or invalid outcome information (i.e. no available folic acid value), people who were not Queensland residents according to their postcode and those with missing or invalid information on gender and age were excluded from analysis. Regarding completeness, a quality criterion of $90 \%$ was set. This criterion was not satisfied for the years 2000-2003 and thus these data were excluded from statistical analysis (Fig. 1).

The necessity for routine folic acid measurement is usually determined by the clinician responsible for diagnosis and medical treatment of the individual. Our data contained no information regarding test indications or the clinical decisions leading to testing. The study population thus consists of Queensland residents with a routine folic acid measurement during the study period.

\section{Folic acid measurement and endpoints}

Folic acid values were investigated as erythrocyte folate or serum folate during the observation period. Sample collection, transportation and processing were conducted under routine clinical conditions, with analysis performed by accredited Queensland pathology services. These processes were likely to assure high sample quality and validity of the test results. Due to the long study observation period, multiple assay changes occurred within laboratories. Folic acid values were therefore analysed mainly as dichotomous variables to assess folic acid deficiency (deficient $v$. non-deficient) based on the cut-off values of assays. For the time period 2008-2010 (i.e. adjacent to the implementation of the mandatory fortification programme in 2009), erythrocyte folate was consistently measured by the Folate Plasma and Serum Enzyme Immunoassay Beckman Coulter Chemical Pathology assay with a lower erythrocyte folate reference limit of $356 \mathrm{nmol} / \mathrm{l}$. Therefore, quantitative analyses of folic acid values were possible for this time 
period. A recent review raised concerns regarding the comparability of studies on folic acid deficiency when different assays and cut-off values are being compared ${ }^{(18)}$. In order to compare different studies, the authors introduced specific factors for assays, cut-off and also a 'prevalence factor'. They found assays to give 'likely correct' estimates if the prevalence factor was between 0.85 and 1.15 . The prevalence factor for erythrocyte folate measured by the Beckman Coulter assay was $0 \cdot 89$, so prevalence measures could be regarded as 'likely correct' based on these considerations.

The WHO recommends a cut-off value of $906 \mathrm{nmol} / \mathrm{l}$ for the detection of folic acid insufficiency in women of childbearing age ${ }^{(17)}$. This cut-off value was applied for this purpose in the subgroup of women of childbearing age in the current analysis to assess folic acid insufficiency.

\section{Statistical analysis}

Due to several changes in the test method, folate measurements over the whole observation period from 2004 to 2015 were analysed as binary information (deficient $v$. non-deficient and insufficient $v$. non-insufficient, respectively). The status of 'folate deficiency' was determined separately for each individual based on the test method used and the respective cut-off value for this specific test. Quantitative analysis of folic acid values was conducted for the years 2008-2010 as there were no test changes during this period. Please note that the term 'prevalence' of folate deficiency is used throughout the present paper for describing the proportion of people with low folic acid levels out of those routinely tested for folic acid during the study period. The stated prevalence thus should not be used as an estimate of overall population prevalence since those tested may be a selected group.

The distribution of continuous variables was assessed and due to skewed distributions in all continuous variables, medians and interquartile ranges (IQR) are reported. Nonparametric test methods were applied for statistical testing. The risk for the occurrence of folic acid deficiency for Indigenous compared with non-Indigenous people was calculated as the risk ratio of proportions in the respective groups. The $95 \% \mathrm{CI}$ for risk ratios were calculated based on the method proposed by Miettinen and Nurminen ${ }^{(19)}$.

Additional multivariate logistic regression analyses were conducted for the crucial time period of 2008-2011 adjacent to the introduction in 2009 to assess whether the observed substantial decline in folic acid deficiency after the mandatory fortification was confounded by demographic variables. To this end, the crude OR (including 95\% CI) for folic acid deficiency between 2010 and $2011 v$. 2008 (as the baseline) and the adjusted OR (adjusted for ethnicity, Indigenous status, gender and age) were calculated.

All analyses were conducted with the statistical software package IBM SPSS Statistics version 25. For all statistical tests, the $\alpha$ level was set to $0 \cdot 05$.

\section{Ethical considerations}

The study was approved by the Townsville Hospital and Health Service Human Research Ethics Committee (number HREC/16/QTHS/15) and also received approval under the Queensland Public Health Act for waiver of consent in the use of identifiable or potentially re-identifiable confidential health information (number RD006385).

\section{Results}

\section{Study population}

In total 291908 initial measurements of folic acid between 1 January 2004 until 31 December 2015 were analysed (see exclusion criteria above). Of these, $53.9 \%(n 157286)$ were from women and the median age at measurement was 60 (IQR 40-77) years. The majority of measurements $(84.7 \%, n 247301)$ were from non-Indigenous people, $4.0 \%$ ( $n 11622$ ) were from Aboriginal people, $0.8 \%$ ( $n$ 2299) were from Torres Strait Islander people and $0 \cdot 3 \%$ ( $n 871$ ) were from people who identified as both Aboriginal and Torres Strait Islander. No time trend in demographics was observed (see online supplementary material, Supplemental Table S1). Ethnicity was missing in $2.7 \%$ of all cases $(n 7852)$ and 'not stated' in $7.5 \%$ ( $n 21$ 963); missing values occurred mainly before 2007.

\section{Prevalence of folic acid deficiency}

The overall prevalence of folic acid deficiency in the studied population of routine folic acid measurements was $3.0 \%$ ( $n 8833)$; it was $7.5 \%$ ( $n 5714$ ) over the time period before mandatory folic acid fortification (2004-2008) v. $1 \cdot 1 \%$ ( $n$ 1985) after the introduction of fortification (2010-2015). Overall, this indicates a relative reduction of $85 \%(P<0 \cdot 001)$.

For Indigenous people, the relative reduction of folic acid deficiency was $93 \%$ with a prevalence of $17.4 \%$ before and $1.3 \%$ after $2009(P<0.001)$. In the nonIndigenous population, a relative reduction of folic acid deficiency of $84 \%$ was observed with a prevalence of $7 \cdot 0 \%$ ( $n$ 4437) before and $1 \cdot 1 \%(n 1721)$ after introduction of mandatory folic acid fortification $(P<0.001)$. The relative reduction of folic acid deficiency was $88 \%$ for women, $7.3 \%$ ( $n$ 2964) before and 0.9\% ( $n$ 945) after mandatory folic acid fortification $(P<0.001)$, and $84 \%$ for men, $7.8 \%(n 2750)$ before and 1.2\% ( $n$ 1040) after 2009 $(P<0.001)$. In the subgroup of women of childbearing age, the prevalence of folic acid deficiency for Indigenous women was $15.9 \%(n 380)$ before and $1.2 \%$ ( $n 65)$ after 2009 , resulting in a risk reduction of $93 \%$ $(P<0.001)$. For non-Indigenous women of childbearing age, the reduction was $85 \%$ with a prevalence of folic acid deficiency of $6.7 \%$ ( $n 2230)$ before and $1.0 \%$ after $2009(P<0 \cdot 001)$. 
The prevalence of folic acid deficiency over the years for the total population and for subgroups by gender, Indigenous status and for the special subgroup of women of childbearing age are detailed in Table 1. For women of childbearing age, the prevalence of folic acid insufficiency during the implementation of the mandatory fortification programme was also assessed. Along with a decrease in the prevalence of folic acid deficiency, folic acid insufficiency decreased likewise with a prevalence of $57.7 \%$ in 2008 (8849/15329), $56.6 \%$ in 2009 (8975/ $15851)$ and $38.8 \%$ in $2010(6331 / 16304)$. Folic acid insufficiency occurred more often in Indigenous women (90.1\% (263/292) in 2008, 88.0 \% (292/332) in 2009 and $65.1 \%$ (237/ $364)$ in 2010) than in non-Indigenous women of childbearing age $(66.6 \%(1517 / 2277)$ in $2008,66.1 \%$ (1416/ $2142)$ in 2009 and $43.6 \%(983 / 2253)$ in 2010).

The prevalence of folic acid deficiency was higher in Indigenous people compared with non-Indigenous people from 2004 until 2010. After 2010 the proportion of folic acid deficiency was comparable between Indigenous and non-Indigenous people (Figs 2 and 3).

\section{Quantitative analysis of erythrocyte folate values}

An analysis of the quantitative folic acid tests was conducted for the years 2008-2010, a time period when test assays and target parameters remained constant. During this time period folate measurements from 88012 people were analysed, with $98.9 \%$ ( $n 87081$ ) based on erythrocyte folate. Overall, the median erythrocyte folate value was 900 (IQR 650-1260) nmol/l. The median erythrocyte folate value increased significantly from 2008 (820 (IQR 580-1180) nmol/l) over 2009 (840 (IQR 610$1200) \mathrm{nmol} / \mathrm{l}$ ) to 2010 (1020 (IQR 780-1350) nmol/l; $P<0.001$; Table 2). This increase was also evident in subgroups by gender, Indigenous status and women of childbearing age (Table 2).

\section{Logistic regression analysis}

Logistic regression analysis was done to assess whether the observed substantial decline in folic acid deficiency (directly before $v$. directly after the mandatory fortification) was confounded by demographic variables. The analysis was based on a total of 88350 cases and revealed crude OR for folic acid deficiency of 0.21 (95\% CI $0 \cdot 18$, $0.23 ; P<0.001)$ for 2010 v. 2008, and 0.37 (95\% CI $0.34,0.41 ; P<0.001)$ for $2011 v$. 2008. The respective adjusted OR (adjusted for ethnicity, gender and age) for folic acid deficiency were 0.20 (95\% CI $0.18,0.23$; $P<0.001$ ) for $2008 v$. 2010, and 0.38 (95\% CI 0.35 , $0.42 ; P<0.001)$ for $2011 v$. 2008. Since the adjusted OR were virtually identical to the crude OR in this subset of data, it is unlikely that changing demographic factors significantly impact the results demonstrated for the entire study period.

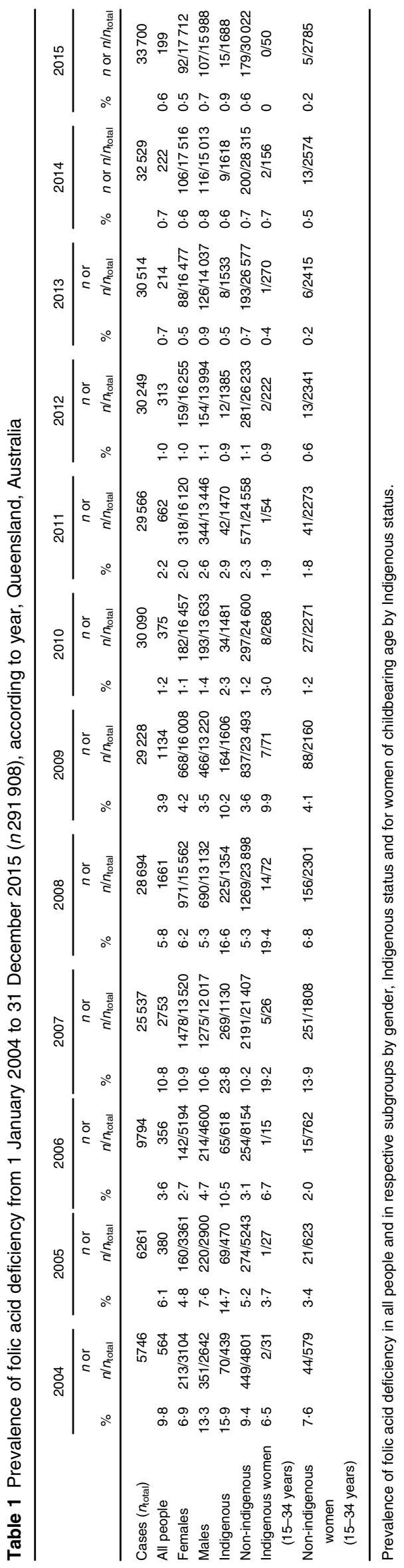




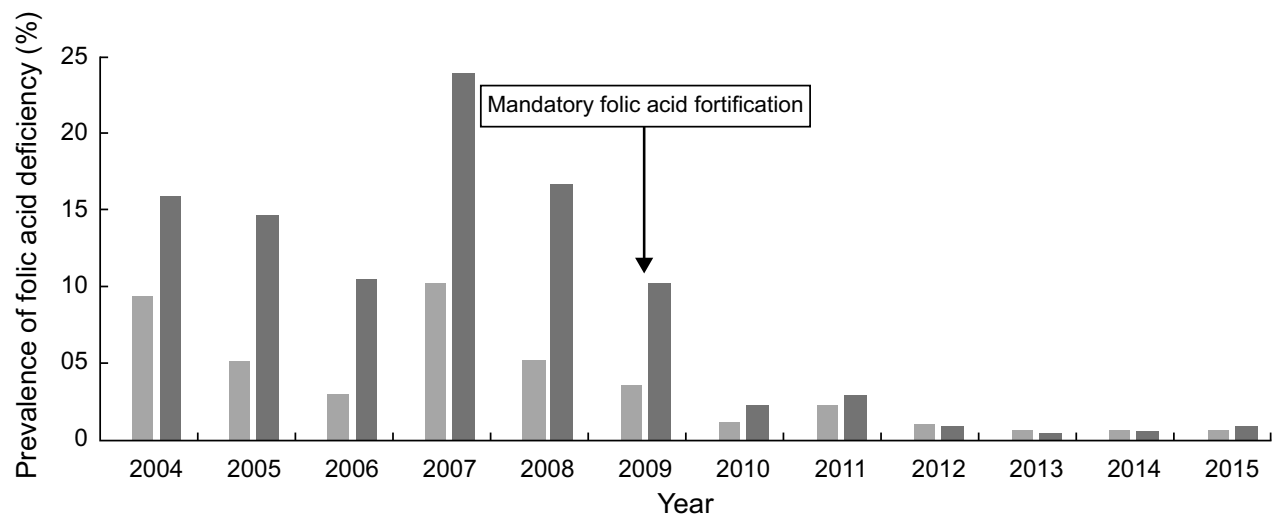

Fig. 2 Prevalence of folic acid deficiency in a sample of routine folic acid measurements ( $n 291908$ ) from 1 January 2004 to 31 December 2015, according to Indigenous status (๓, non-Indigenous; $₫$, Indigenous), Queensland, Australia

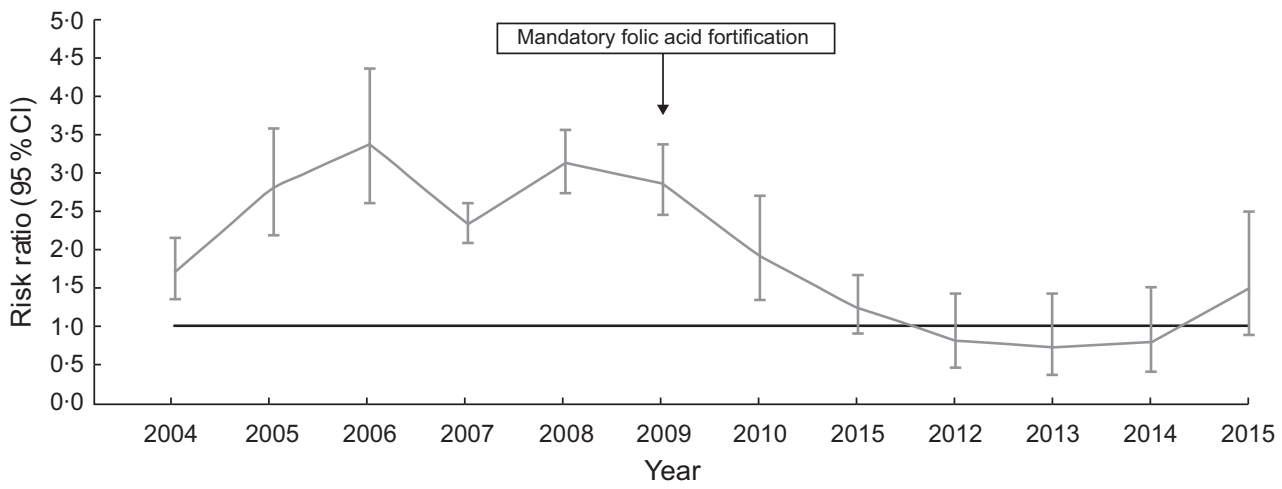

Fig. 3 Unadjusted risk ratios, with $95 \% \mathrm{Cl}$ represented by vertical bars, for the association between folic acid deficiency and Indigenous status, from 1 January 2004 to 31 December 2015 (n291 908), Queensland, Australia: —, risk ratio between the prevalence of folic acid deficiency in Indigenous $v$. non-Indigenous persons; - , reference line included to illustrate a risk ratio of 1 (identical risk in both groups)

\section{Discussion}

The present study is the first to shed light not only on the status quo of folic acid deficiency before mandatory fortification, but more importantly also on the impact of the mandatory folic acid fortification programme in Australia in a large-scale, state-wide population. Moreover, it is also the first study to evaluate this programme in a large, unselected Indigenous population and in other specific high-risk groups.

Despite the introduction of voluntary folic acid fortification in 1995, the present study revealed a very high prevalence of folic acid deficiency before implementation of the mandatory folic acid fortification programme in 2009. However, after 2009, a substantial and statistically significant decline of folic acid deficiency was observed for the overall population, for Indigenous and non-Indigenous people, and also for women of childbearing age. The observed decline was unlikely to be confounded by changing demographic characteristics in the study population over the observation period.
The observed relative reductions of folic acid deficiency in the range of $80-90 \%$ provide positive evidence that mandatory folic acid fortification in Queensland has been successful, even for people in high-risk populations.

\section{Evaluation of voluntary folic acid fortification}

The voluntary folic acid fortification programme of flour, savoury biscuits, bread, breakfast cereals, pasta, rice, yeast extracts as well as fruit and vegetable juices was implemented in 1995 in Australia ${ }^{(2)}$ and led to a decline in the prevalence of folic acid deficiency from $8.5 \%$ in the years $1993-1996$ to $4 \cdot 1 \%$ in 2000 in a population of 20506 women and men aged 14-45 years in the Melbourne city area ${ }^{(3)}$. Halliday and Riley observed a decline in NTD in newborns over the years 1996-1999 in the state of Victoria ${ }^{(4)}$. These findings were underlined by an investigation in South Australia regarding the years 1966-2007 showing an increased knowledge on the importance of folic acid intake in pregnant women as well as a decline of NTD from 2.06 per 1000 births in 1986-1990 to 1.23 per 1000 births in $2002-2007^{(5)}$. 
Despite these encouraging effects, other investigations highlighted the inequities regarding the impact of this primary health-care measure in high-risk populations.

The National Nutrition Survey in 1995 and 2007 revealed that tailored interventions are needed to reach all subgroups of the target population, particularly in groups with low socio-economic status and women of childbearing age $^{(16)}$. A cross-sectional study of 424 Aboriginal and 232 Torres Strait Islander women aged 15-34 years in twenty-three rural and remote communities in far north Queensland showed a high prevalence of folic acid deficiency in women of childbearing age (Aboriginal $32 \%$, Torres Strait Islander $16 \%)^{(20)}$. These findings might be explained by a low health literacy of Indigenous women of childbearing age regarding the role of folic acid in pregnancy, especially in teenage mothers ${ }^{(7)}$. Accordingly, the investigation of trends in NTD in Western Australia showed no decline for Indigenous women between 1980 and 2000, with rates of 2.55 NTD per 1000 in live and stillbirths between 1980 and 1992, and 2.56 NTD per 1000 births between 1996 and $2000^{(7)}$. These findings are consistent with the present investigation for the time period before mandatory folic acid fortification (2004-2008), where we observed a high prevalence of folic acid deficiency both in the overall general population and in the Indigenous sub-population.

\section{Evaluation of mandatory folic acid fortification}

According to a recent review on folic acid, a reference daily intake of $400 \mu \mathrm{g} / \mathrm{d}$ during the periconception period is recommended in most countries worldwide and by the WHO. Correspondingly in Australia folic acid intake of $400 \mu \mathrm{g} / \mathrm{d}$ is recommended for non-pregnant women and $600 \mu \mathrm{g} / \mathrm{d}$ for pregnant women ${ }^{(21)}$. According to the Australian Food Standards, "wheat flour that is sold as suitable for making bread' should contain $2-3 \mathrm{mg}$ folic acid/kg since September $2009^{(12)}$. This recommendation should then assure a folic acid intake of $120 \mu \mathrm{g}$ per $100 \mathrm{~g}$ bread consumed $^{(13)}$. A comparable mandatory fortification programme was introduced in the USA in 1998 with a folic acid fortification policy of $140 \mu \mathrm{g} / 100 \mathrm{~g}$ grain product. This programme resulted in a mean increase of erythrocyte folate values from 747 to $1120 \mathrm{nmol} / \mathrm{l}$ based on National Health and Nutrition Examination Survey data and a $31 \%$ reduction in $\mathrm{NTD}^{(1)}$. Mean erythrocyte folate values in the USA increased by $57 \%$ from 375 to $590 \mathrm{nmol} / 1$ while the prevalence of low erythrocyte as well as serum folate values decreased ${ }^{(6,22)}$. Simultaneously NTD declined by $19 \%$ after mandatory folic acid fortification ${ }^{(23)}$. Studies from the USA not only provided evidence that mandatory fortification programmes could be successful, but also revealed a higher prevalence of folic acid deficiency in specific ethnic groups such as non-Hispanic Black women as compared with non-Hispanic White and Mexican-American women $^{(24)}$. 
Mandatory folic acid fortification in Australia resulted in an estimated folic acid intake of $159 \mu \mathrm{g} / \mathrm{d}$ based on the analysis of 100 breads with a mean folic acid concentration of $200 \mu \mathrm{g} / 100 \mathrm{~g}$ bread in June/July 2010. This estimate exceeded the intended amount by $80 \mu \mathrm{g} / 100 \mathrm{~g}^{b^{2} e a d^{(25)}}$.

The largest cohort in Australia to date investigated blood folate levels in 20592 blood samples from a diagnostic pathology laboratory in Sydney ${ }^{(15)}$. A decrease of $85 \%$ from 3.4 to $0.5 \%$ of low erythrocyte folate values between April 2009 and April 2010 was reported, while mean erythrocyte folate increased from 881 to $1071 \mathrm{nmol} / \mathrm{l}$ during the same time period ${ }^{(15)}$. These findings are consistent with our analysis of 291908 folic acid measurements in Queensland between 2004 and 2015, where we found the prevalence of folic acid values below the reference limit was $9.4 \%$ in 2004 and declined to $0.6 \%$ in 2015. In the years 2009 and 2010 a decline from 3.9 to $1.2 \%$ prevalence of low folic acid values was observed. Regarding the impact of the mandatory fortification programme in Indigenous populations, a benefit of the mandatory fortification programme was shown in a sample of ninety-five Aboriginal men and non-pregnant Aboriginal women aged 16-44 years in urban and regional Western Australia ${ }^{(14)}$. A dietary assessment was conducted along with erythrocyte folate measurements between November 2013 and January 2014 for all participants and folic acid values were compared with values observed in a previous study. The authors were able to show a mean increase in erythrocyte folate values for males $(129 \mathrm{ng} / \mathrm{ml})$ and females $(186 \mathrm{ng} / \mathrm{ml})$, and no values below the reference limit were observed. This coincided with a decline in NTD of $68 \%$ in Aboriginal infants in Western Australia based on information from the Western Australia Register of Developmental Anomalies ${ }^{(14)}$. The current investigation of Queenslandwide data confirmed this finding in a larger Indigenous population of 14792 individuals. The prevalence of folic acid deficiency declined by $86 \%$ from $16.6 \%$ in 2008 to $2.3 \%$ in 2010. These findings also suggest that the Indigenous population in Queensland benefited from the mandatory fortification programme to a greater extent than the nonIndigenous population, in whom the prevalence of folic acid deficiency decreased by $77 \%$ from 5.3 to $1.2 \%$ for the same time period. This pattern is comparable to a prevalence observed in an Aboriginal sample of 191 individuals in 2008-2009 from two regional and two metropolitan sites in Perth ${ }^{(26)}$. Ten per cent of Aboriginal women and $26 \%$ of Aboriginal men were folic acid deficient in that investigation. We also found that the subgroup of Indigenous women of childbearing age (15-34 years) with the highest prevalence of folic acid deficiency in 2008 benefited most: the prevalence of folic acid deficiency declined by $84 \%$ from $19.2 \%$ in 2008 to $3.0 \%$ in 2010 . These effects are comparable to recently reported data from Tanzania; after the implementation of mandatory folic acid fortification the prevalence of folic acid deficiency declined from
$26.9 \%$ at baseline to $5 \%$ after 12 months ${ }^{(27)}$. Additionally, this reduction of folic acid deficiency was mirrored by a decline in NTD when the periods before and after the implementation of a mandatory folic acid fortification programme were compared ${ }^{(28)}$. For Queensland the NTD per 10000 births declined from 14.6 in 2007 to 10.0 in 2011 . A significant reduction was also observed in teenage mothers and Indigenous residents in the Australian states and territories of New South Wales, Queensland, Western Australia, South Australia and Northern Territory. The NTD rate per 10000 conceptions for Indigenous residents reduced from 19.6 at baseline to 5.1 after mandatory folic acid fortification $^{(28)}$. A comparable decline in NTD after implementation of mandatory folic acid fortification of staple foods has also been observed in South Africa where overall NTD declined by $30.5 \%$, NTD perinatal deaths declined by $65.9 \%$ and NTD infant mortality declined by $38 \cdot 8 \%{ }^{(29)}$.

\section{Folic acid insufficiency}

Although folic acid deficiency as well as NTD declined, our study found high prevalence of folic acid insufficiency in women of childbearing age with unknown pregnancy status. According to a WHO recommendation, erythrocyte folate concentrations below $906 \mathrm{nmol} / 1$ should be avoided in non-pregnant women of childbearing age ${ }^{(17)}$. Further monitoring is suggested to assure adequate folic acid levels in non-pregnant women of childbearing age and especially those with an Indigenous background, to avoid folic acid insufficiency and the associated occurrence of NTD.

\section{Limitations}

The current analysis of routinely available data from pathology laboratories in Queensland has some limitations that need to be taken in consideration regarding the interpretation of results.

Any research based on routinely available health data is limited by the quality of the available data. In the present investigation data had to be excluded due to incompleteness and the occurrence of invalid values.

Recently concerns have been raised regarding the comparability of prevalences of folic acid deficiency when different assays are being utilised for erythrocyte folate measurement ${ }^{(18)}$. During the implementation of the mandatory folic acid fortification an assay with a high comparability to the current gold standard measurement technique was used and thus prevalences are likely comparable during this time period. The current analysis, however, is still the first large-scale evaluation of the mandatory folic acid fortification programme in Australia. Further studies should investigate other states and should, whenever possible, take assay changes into account when prevalences are being compared.

Moreover, the present study is the first state-wide evaluation of folic acid values across Queensland and it 
has to be recognised that our data might not be considered a representative sample of the whole Queensland general population. Especially regarding the findings in Indigenous sub-populations, the Queensland Indigenous population might differ from other Indigenous communities regarding social, cultural or environmental determinants of health and thus differences might also apply for folic acid values and the impact of the mandatory fortification programme. Our study comprises a sample of clinical folic acid measurements and might thus be biased towards a higher prevalence of folic acid deficiency. It is therefore not representative of the general community, but rather a sub-population of people seeking health services, and therefore again potentially biased toward lower folate values. However, there is no reason to assume that this selection bias changed during the period under investigation, so before-and-after comparison should remain valid. Furthermore, folic acid levels could be impacted by the intake of certain drugs, pregnancy status, folic acid supplementation and co-morbidities. Since no information about current prescriptions and drug intake, pregnancy status, folic acid supplementation and co-morbidities was available in the current study, no dedicated analyses regarding these subgroups were possible. Concerning the high number of analysed individuals and the exclusion of repeated measurements, it seems rather unlikely that the overall measurements are biased by this limitation regarding data availability. Future dedicated studies should confirm our results in prospective data collections concerning also the abovementioned influencing factors.

Another limitation of the present study is that nutritional behaviour changes over time and may be a factor in the observed decline in folic acid deficiency and independent of the folic acid fortification programme. Due to a lack of food intake data it thus remains unclear whether, and to what extent, the decline in folic acid deficiency is attributable to the mandatory fortification programme.

While mandatory folate fortification appears to have a big impact on folate levels and seems successful in reaching high-risk populations, poor overall nutrition and low health literacy are major determinants of adverse health outcomes and further policy interventions need to be considered and evaluated $^{(8,9,20,28,30,31)}$.

\section{Conclusion}

The mandatory fortification of bread flour with folate appears to have had a significant positive impact on folate levels across the whole population in Queensland, especially benefiting high-risk groups which the voluntary programme failed to reach. However, these high-risk groups may remain poorly nourished overall and single nutrient supplementation could be insufficient.

\section{Acknowledgements}

Financial support: This study was funded by Queensland Health (Funding ID: SCRF-Round 3. Funding type: Senior Clinical Research Fellowship. Term: 5 years; commencement date 22 October 2012, extension was granted for a further 12 months). Queensland Health had no role in the design, analysis or writing of this article. Conflict of interest: All authors declare that they do not have any study-related conflicts of interest. Authorship: R.McD. designed the research project; L.H., S.C. and R.McD. obtained ethics approval and data from Pathology Queensland; A.S. prepared the data for statistical analyses; and A.S. and R.M. performed all statistical analyses. A.S. drafted the paper. A.S., R.M. and R.McD. have primary responsibility for the final content of the paper. All authors have contributed to interpretation of data and manuscript development. Ethics of buman subject participation: This study was conducted according to the guidelines laid down in the Declaration of Helsinki and all procedures involving human subjects were approved by the Townsville Hospital and Health Service Human Research Ethics Committee (number HREC/16/QTHS/15) and also received Public Health Approval for waiver of consent in the use of identifiable or potentially re-identifiable confidential health information (number RD006385).

\section{Supplementary material}

To view supplementary material for this article, please visit https://doi.org/10.1017/S1368980019002258

\section{References}

1. Choi JH, Yates Z, Veysey M et al. (2014) Contemporary issues surrounding folic acid fortification initiatives. Prev Nutr Food Sci 19, 247-260.

2. Adams T \& Jeffreson S (1996) Australia implements voluntary folate fortification. Am J Public Health 86, 593-594.

3. Metz J, Sikaris KA, Maxwell EL et al. (2002) Changes in serum folate concentrations following voluntary food fortification in Australia. Med J Aust 176, 90-91.

4. Halliday JL \& Riley M (2000) Fortification of foods with folic acid. N Engl J Med 343, 970-971; author reply 972.

5. Chan AC, van Essen P, Scott H et al. (2008) Folate awareness and the prevalence of neural tube defects in South Australia, 1966-2007. Med J Aust 189, 566-569.

6. Lawrence JM, Petitti DB, Watkins M et al. (2000) Trends in serum folate after food fortification. Lancet 354, 915-916.

7. Bower C, Eades S, Payne J et al. (2004) Trends in neural tube defects in Western Australia in Indigenous and nonIndigenous populations. Paediatr Perinat Epidemiol 18, 277-280.

8. D'Onise K, McDermott RA, Leonard D et al. (2012) Lack of folate improvement in high risk indigenous Australian adults over an average of 6.5 years: a cohort study. Asia Pac J Clin Nutr 21, 431-439. 
9. Gall S, Seal J, Taylor R et al. (2012) Folate status and sociodemographic predictors of folate status, among a national cohort of women aged 26-36 in Australia, 2004-2006. Aust N Z J Public Health 36, 421-426.

10. Maberly GF \& Stanley FJ (2005) Mandatory fortification of flour with folic acid: an overdue public health opportunity. Med J Aust 183, 342-343.

11. Ekert H (2006) Mandatory fortification of flour with folic acid: an overdue public health opportunity. Med J Aust 184, 199; author reply 199 .

12. Federal Register of Legislation, Australian Government (2015) Australia New Zealand Food Standards Code Standard 2.1.1 - Cereal and cereal products. https://www. legislation.gov.au/Details/F2015L00420 (accessed May 2018).

13. Food Standard Australia New Zealand (2015) Mandatory Fortification of Bread with Folic Acid and Iodine. https:// www.foodstandards.gov.au/consumer/nutrition/folicmandat ory/documents/Mandatory\%20Fortification\%20Brochure $\% 20$ for\%20Health\%20Professionals.pdf (accessed May 2018).

14. Bower C, Maxwell S, Hickling S et al. (2016) Folate status in Aboriginal people before and after mandatory fortification of flour for bread-making in Australia. Aust $N Z J$ Obstet Gynaecol 56, 233-237.

15. Brown RD, Langshaw MR, Uhr EJ et al. (2011) The impact of mandatory fortification of flour with folic acid on the blood folate levels of an Australian population. Med J Aust 194, 65-67.

16. Emmett JK, Lawrence M \& Riley M (2011) Estimating the impact of mandatory folic acid fortification on the folic acid intake of Australian women of childbearing age. Aust $N Z \mathrm{~J}$ Public Health 35, 442-450.

17. Cordero AM, Crider KS, Rogers LM et al. (2015) Optimal serum and red blood cell folate concentrations in women of reproductive age for prevention of neural tube defects: World Health Organization guidelines. MMWR Morb Mortal Wkly 64, 421-423.

18. Rogers LM, Cordero AM, Pfeiffer CM et al. (2018) Global folate status in women of reproductive age: a systematic review with emphasis on methodological issues. Ann NY Acad Sci 1431, 35-57.

19. Miettinen ON \& Nurminen M (1985) Comparative analysis of two rates. Stat Med 4, 213-226.

20. McDermott R, Campbell S, Li M et al. (2009) The health and nutrition of young indigenous women in north Queensland intergenerational implications of poor food quality, obesity, diabetes, tobacco smoking and alcohol use. Public Health Nutr 12, 2143-2149.
21. Gomes S, Lopes C \& Pinto E (2016) Folate and folic acid in the periconceptional period: recommendations from official health organizations in thirty-six countries worldwide and WHO. Public Health Nutr 19, 176-189.

22. Dietrich M, Brown CJP \& Block G (2005) The effect of folate fortification of cereal-grain products on blood folate status, dietary folate intake, and dietary folate sources among adult non-supplement users in the United States. J Am Coll Nutr 24, 266-274.

23. Honein MA, Paulozzi LJ, Mathews TJ et al. (2001) Impact of folic acid fortification of the US food supply on the occurrence of neural tube defects. JAMA 285, 2981-2986.

24. McDowell MAL, David A \& Pfeiffer CM (2008) Blood Folate Levels: The Latest NHANES Results. NCHS Data Brief no. 6. Hyattsville, MD: National Center for Health Statistics; available at https://www.cdc.gov/nchs/data/databriefs/db 06.pdf (accessed July 2019).

25. Dugbaza J \& Cunningham J (2012) Estimates of total dietary folic acid intake in the Australian population following mandatory folic acid fortification of bread. J Nutr Metab 2012, 492353.

26. Maxwell SJ, Brameld KJ, Bower C et al. (2013) Baseline investigations of folate status in Aboriginal and non-Aboriginal West Australians prior to the introduction of mandatory fortification. Aust N Z J Obstet Gynaecol 53, 26-31.

27. Noor RA, Abioye AI, Ulenga N et al. (2017) Large-scale wheat flour folic acid fortification program increases plasma folate levels among women of reproductive age in urban Tanzania. PLoS One 12, e0182099.

28. Morris N, Stewart S, Riley M et al. (2018) Differential impact of malnutrition on health outcomes among indigenous and non-indigenous adults admitted to hospital in regional Australia - a prospective cohort study. Nutrients 10, 644 .

29. Sayed AR, Bourne D, Pattinson R et al. (2008) Decline in the prevalence of neural tube defects following folic acid fortification and its cost-benefit in South Africa. Birth Defects Res A Clin Mol Teratol 82, 211-216.

30. Lee YQ, Collins CE, Gordon A et al. (2018) Disparities exist between the dietary intake of Indigenous Australian women during pregnancy and the Australian dietary guidelines: the Gomeroi gaaynggal study. J Hum Nutr Diet 31, 473-485.

31. Morris NF, Stewart S, Riley MD et al. (2018) The burden and nature of malnutrition among patients in regional hospital settings: a cross-sectional survey. Clin Nutr ESPEN 23, 1-9. 